\title{
Regenerative potential of secretome from dental stem cells: a systematic review of preclinical studies
}

\begin{abstract}
Injury to tissues is a major clinical challenge due to the limited regenerative capacity of endogenous cells. Stem cell therapy is evolving rapidly as an alternative for tissue regeneration. However, increasing evidence suggests that the regenerative ability of stem cells is mainly mediated by paracrine actions of secretome that are generally secreted by the cells. We aimed to systematically evaluate the efficacy of dental stem cell (DSC)-conditioned medium in in vivo animal models of various tissue defects. A total of 15 eligible studies was included by searching Pubmed, Scopus and Medline databases up to August 2017. The risk of bias was assessed using the Systematic Review Centre for Laboratory Animal Experimentation risk of bias tool. Of 15 studies, seven reported the therapeutic benefit of the conditioned medium on neurological diseases and three reported on joint/bone-related defects. Two interventions were on liver diseases, whereas the remaining three addressed myocardial infarction and reperfusion, lung injury and diabetes. Nine studies were performed using mouse models and the remaining six studies used rat models. The methodological quality of the studies was low, as most of the key elements required in reports of preclinical studies were not reported. The findings of this review suggested that conditioned medium from DSCs improved tissue regeneration and functional recovery. This current review strengthens the therapeutic benefit of cell-free product for tissue repair in animal models. A well-planned study utilizing validated outcome measures and long-term safety studies are required for possible translation to clinical trials.
\end{abstract}

Keyword: Conditioned medium; Dental stem cells; Secretome; Tissue defect; Tissue regeneration 